\title{
Design of Interface Programs for Power Quality Standard Device
}

\author{
Lei Shao ${ }^{1}$, Xiang Jing ${ }^{2}$ and Zhong Chen ${ }^{1}$ \\ ${ }^{1}$ School of Electrical Engineering, Southeast University, Nanjing, China \\ ${ }^{2}$ You Dian Technologies Limited Company, Wuxi, China
}

\begin{abstract}
This paper analyses the status of automatic test technology and the testing of power quality monitoring devices, and a set of power quality standard source controlled interface program is developed according to the characteristics and requirements of test performance. Based on the integrated development platform of Visual Studio 2008, the program is hierarchical and modular designed, the software architecture of test system is built. The test terminal completes the processes of information collection, measurement, controlling and detection of various test commands. And the test terminal generates the standardized test report automatically, achieves one button-style convenient control completely, finish the power quality automated testing. The interface program is able to access to the automatic test system well, can improve the efficiency and guarantee the quality in practical application of testing process.
\end{abstract}

Keywords-power quality; automatic test; interface programs; standard data interface

\section{INTRODUCTION}

As the improvement of automation and intelligent degree of the substation, test application service interface as an indispensable part of measurement and automation system, automatic test system in the design of interface programs are varied due to their diversity in interface design and control software. At present, most of the power quality monitoring devices on the market are single loop devices, disadvantages are growing for testing method is given priority to with manual testing: tedious and inefficiently process, low reliability, without unified formation testing data [1]-[2]. On the other hand, the compatibility between the various instruments and test system is very poor duo to the large differences of equipment in control software and interface designed by different manufacturers [3]-[5].

In this paper, a set of interface program for power quality standard device control is developed combined with the research of automatic test technology and system. Criterions of software invoking in interface programs are established by using COM component technology; test functions data interface library is also established, with standardized methods of interface for test functions and parameters.

\section{Automatic Test System}

A automatic test system is composed of terminals, tester, and the device under test, the system hardware structure is shown in Figure I. Testing machine or personal PC as control terminal of automatic test system, access to the interchanger, as well as the tester and the device access switches in order to form a communication link, the connection between the tester and the device under test implemented through test lines [6].

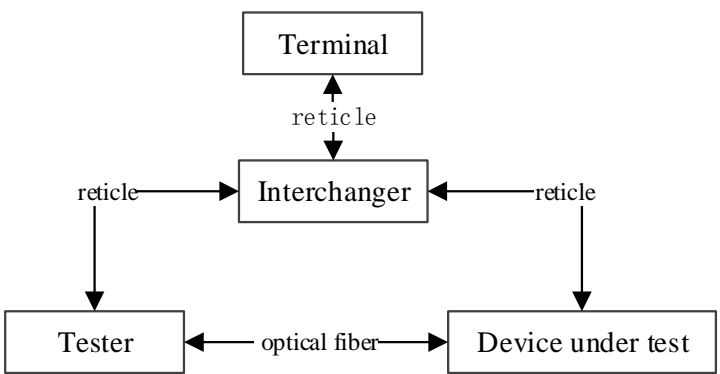

FIGURE I. HARDWARE STRUCTURE OF AUTOMATIC TEST SYSTEM

In order to completely solve carious disadvantages of manual test, test equipment from different manufactures have its own control software and interface, terminal realizes information collection, measurement, control and testing via all sorts of commands and process, and the test results are reported to form a standardized reports, to achieve "one-button" convenient control [7]-[10]. The automatic test system must to do the following:

\section{A. Maintenance of Test Scheme}

A automatic test system needs an efficient test scheme development platform for devices, which is able to quickly edit device testing solutions, complete the automatic test of different kinds of devices conveniently. This platform adopts the mothed of abstracting from the basic principle of testing to abstract the same test functions as test template; it also designs appropriative module for test scheme development, which helps generate test scheme automatically and improve the efficiency.

\section{B. Communication Protocol}

A automatic test system to be able to communicate with the device under test, and realize the data read from the devices under test, modification of the devices' setting, send commands to the devices.

All kinds of commands are designed and edited by devices test scheme subsystem, the automatic test system contains communication block, which is used to execute any communicated commands, and results are returned to control center of the main system, so as to realize the closed-loop automatic test. 


\section{System Expansion}

The diversification from manufacturers requires flexible system architecture, upgrade and extension of functions conveniently, thus, the structure of a automatic test system is to be considered able to support various testers.

Automatic test is designed based on the thought of layered architecture as well as block-structure, data interaction between each module in order to realize the unification of comprehensive control. Interface module specifications of tester control, standard data interface specifications of test functions are established, the control center control the tester according to these two specifications, to realize the functions of Power Quality test automatically.

\section{Standardization and High Efficiency}

The test scheme of automatic test system conforms to standard Power Quality verification regulations, control center in strict accordance with the scheme for automatic testing, records the testing process and results, determines whether the results qualified, fills in a test report in standard format automatically.

\section{Design of Automatic Test System}

\section{A. General Design}

The software architecture of test system conforms to the idea of layered architecture as well as block-structure, as shown in the Figure II. The whole system contains three layers: the interface layer for testers, the automatic test layer and the test scheme development layer.

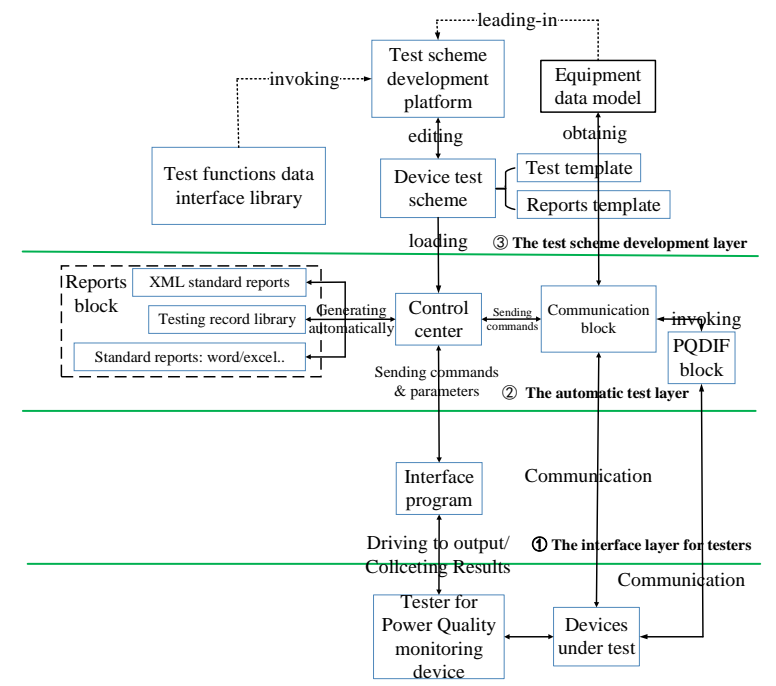

FIGURE II. SOFTWARE ARCHITECTURE OF AUTOMATIC TEST SYSTEM

\section{B. The Interface Layer for Testers}

Interface programs are opened COM components, providing all service interfaces of test functions, which are used for control center. The main task of interface programs is communicating with the system, during the test, the main program sends commands and parameters to interface programs, after then the interface programs drive the tester to output and return the feedback.

\section{The Automatic Test Layer}

This layer is the control center of the whole automatic test system, realize the automatic test of Power Quality monitoring device. The control center provides a GUI, and it opens the test scheme and executes all the processes as defined, determines the results are qualified or not automatically. As the test finished, a report generated in standard format. Standard reports include Word, Excel and Xml format. And the detail information about the test is saved in the test record library: test times, time of each test, test parameters, test results and data read from the device. The communication block in this layer is responsible for communicating with the device under test and returning data to the main program, includes warning and error data.

\section{The Test Scheme Development Layer}

Test scheme is developed to cater different devices and tests in this layer, using device test data model and standard test functions data interface library. The device model is a ICD or SCL document, describes detail information of the device under test. A test scheme includes two parts: test template documents and report template documents. The test template document is based on standard XML language, used for recording the device model and test process. The report template document is a Word document, describes the format of the report and results as well as parameters.

The test functions library including all functions, which are described by function ID, test data and result data, and a function is saved as a standard $\mathrm{Xml}$ format document. Based on the research of Power Quality device, the test function is abstracted as test items and corresponding methods, including test procedures, parameters and results.

\section{Design of InTERface Programs}

\section{A. The Framework of Interface Programs}

Interface programs and are hierarchical and modular designed based on the visual studio 2008 platform using standard C++ language. The structure of interface programs as shown in Figure III.

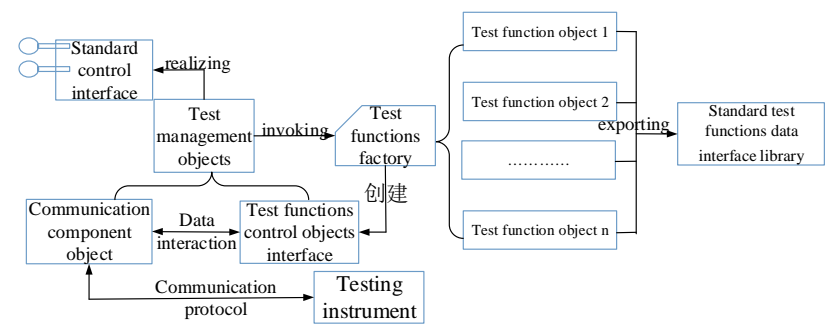

FIGURE III. STRUCTURE OF INTERFACE PROGRAMS

Interface programs contains standard control interface block, communication block and test functions management block. The standard control interface is a COM interface opened to users, it is used to interconnect the tester in the whole test system. The communication block is responsible for communication between tester and interface, including data interaction between tester and test functions management block, as well as communication between test functions 
management block and test management objects block. Test functions management block is divided into test functions library, test functions factory, test functions objects and management objects.

\section{B. Common Interface}

A common interface for testing instrument control is designed, in order to realize tester standard control. The common interface is used to communicate with tester hardware, direct the tester to voltage and current output. Relevant operations during the process of automatic testing are defined, including reading and setting interface configuration parameters, downloading testing parameters, starting and stopping test and loading reports. The control of Power Quality monitoring device is realized in a standardized way through COM component technology.

The automatic test system is able to establish different tester interface programs for different testing instruments produced by different manufacturers. In this way, all interface programs using the same interface specification and test functions data interface specification, communication and control between different testers is realized by unitive client.

\section{Communication Protocol}

Communication block in the system stipulates the interface protocol for data interaction between interface programs and Power Quality devices. Communication specification, the format of control commands and return information are detailed explained in the protocol.

The communication protocol adopts the standard TCP/IP protocol, the Power Quality device is the server while the interface performs as a client. During the process of automatic test, the test system controls all testing processes and the communication block leads all communicating commands, including start test command, stop test command and heartbeat command. The heartbeat command, in order to prevent the disconnections between the test system and Power Quality device, this command is sent to the device every 5 seconds to make sure the information of current state is returned to the system and the connection is normal. And at the same time, the device under test is required to return information to the system to ensure all test processes are correct. Returned information is divided into 3 classes, state information, error information and warning information.

\section{Test Functions Models}

Standard test items and methods are abstracted based on basic principle of Power Quality test, including test processes, test parameters and test results. And on the basis of these data, the unified test functions data interface library is designed, using the technology of XML. Each test function is described by test function ID, test data and result data. Test functions including voltage and current test, harmonic test, inter-harmonic test, flicker test and transient test.

\section{THE SPECIFIC APPLICATION}

After interface programs being designed, connecting the interface and automatic test system to verify the correctness, reliability and practicability of the interface, divided into three steps:
First, testing template is edited by using testing solution development platform, to form a test scheme according to all testing requirements. Second, creating test environment, based on the connections of system hardware, using internet to connect terminals, switches, devices under test and Power Quality testers, to form a testing closed-loop. Finally, automatic testing, the automatic test system opens the test template, clicks the start test button, the test performed as defined. After this, the result is recorded automatically, and a standardized report will be returned.

\section{CONCLUSION}

In this paper, the automatic test technology of Power Quality monitoring device is researched, based on the study of basic principle of Power Quality test, interface programs are designed to achieve the automatic test of Power Quality device in a closed-loop way. The interface program mainly has the following features:

- A set of standardized method for controlling Power Quality monitoring device tester is realized. A general COM interface for tester control software based on the analysis of all kinds of test instruments' working principles, combined with the standard test functions data interface library.

- A standardized method of Power Quality test functions is designed. Standard test items and methods including test processes, test parameters and test results are abstracted, a unified test functions data interface library is designed, this library define all test functions, which are described by test ID, test data and test result.

\section{REFERENCE}

[1] Ying, Z. H., Hu, J. B. and Zhao, R. D. (2010) Research and Design of Relay Protection Equipment Automated Test System. Power System Protection and Control, 38, 142-146.

[2] Zhao, Z. P., Jiang, L. S. and Wang, X. (2013) Design of Power Quality Monitoring Device Automatic Detection System. Yunnan Electric Power, 41, 41-45.

[3] Li, Z. A., Shen, Q. R. and Wang, Y. G. (2009) Development of Precise Capacitive Voltage Divider for Optical Voltage Transformer. Automation of Electric Power Systems, 33, 77-80.

[4] Fu, M. J., Liu, H. Y. and Dong, L. C. (2015) Research and Application of Relay Protection Automatic Test System for Smart Substation. Power System Protection and Control, 43, 40-44

[5] Lai, Q., Hua, J. W. and Lv, Y. (2010) Research on General Relay Protection Auto-test System Software. Power System Protection and Control, 38, 90-94.

[6] Wang, Z., Zhang, X. L. and Li, Z. A. (2015) Design of Automatic Test System for Relay Protection Device. Power System Protection and Control, 43, 130-135.

[7] Yang, W. and Huang, W. T. (2012) Software Design and Implementation of General Automatic Test System. Industrial Control Computer, 25, 79-80.

[8] Zhang, X. L., Liu, H. H. and Li, J. Q. (2015) Automatic Test Platform in Smart Substation for Relay Protection. Automation of Electric Power Systems, 39, 91-96.

[9] Liu, W., Zhao, Y. and Shi, G. (2013) One-key Test System for Relay Protection Equipment of Intelligent Substation. Electric Power Automation Equipment, 33, 152-155.

[10] Chen, Z. M., Chen, H. and Chen, W. (2009) Test System for Complete Digital Protective Relay. Electric Power Automation Equipment, 29, 109-113 